\title{
Searching for new yellow symbiotic stars: positive identification of $\operatorname{StH} \alpha 63$
}

\author{
N.O. Baella ${ }^{1,2}$, C.B. Pereira ${ }^{3}$, L.F. Miranda ${ }^{4}$, and A. Alvarez-Candal ${ }^{3}$ \\ 1: Unidad de Astronomía, Instituto Geofísico del Perú, Lima, Perú \\ 2: Departamento de Ciencias, Sección Física, Pontificia Universidad Católica del Perú, \\ Apartado 1761, Lima, Perú \\ 3: Observatório Nacional/MCTI, Rua Gen. José Cristino, 77, 20921-400, Rio de Janeiro, \\ Brazil \\ 4: Instituto de Astrofísica de Andalucía-CSIC, C/ Glorieta de la Astronomía s/n, E-18008 \\ Granada, Spain \\ Email:nobar.baella@gmail.com,claudio@on.br,lfm@iaa.es,alvarez@on.br
}

Submitted to The Astronomical Journal

Received —

Submitted to the Astronomical Journal

\footnotetext{
${ }^{1}$ Based on the observations collected at the Centro Astronómico Hispano-Alemán, Calar Alto, jointly operated by the Max-Planck-Institut für Astronomie (Heidelberg) and the Instituto de Astrofísica de Andalucía (CSIC), and at the 4.1m telescope at Cerro Pachón Observatory, Chile.
} 


\begin{abstract}
Yellow symbiotic stars are useful targets to probe whether mass transfer has happened in these binary systems. However, the number of known yellow symbiotic stars is very scarce. We report spectroscopic observations of five candidate yellow symbiotic stars selected by their position in the 2MASS $(\mathrm{J}-\mathrm{H})$ versus $\left(\mathrm{H}-\mathrm{K}_{s}\right)$ diagram and included in some emission-line catalogs. Among the five candidates, only $\mathrm{StH} \alpha 63$ is identified as a new yellow symbiotic star because of its spectrum and its position in the $[\mathrm{TiO}]_{1}-[\mathrm{TiO}]_{2}$ that indicates a K4-K6 spectral type. In addition, the derived electron density $\left(\sim 10^{8.4} \mathrm{~cm}^{-3}\right)$ and several emission line intensity ratios provide further support for that classification. The other four candidates are rejected as symbiotic stars because three of them actually do not show emission lines and the fourth one shows only Balmer emission lines. We also found that the WISE W3-W4 index clearly separates normal K-giants from yellow symbiotic stars and, therefore, can be used as an additional tool to select candidate yellow symbiotic stars.
\end{abstract}

Subject headings: binaries : symbiotic stars - stars : fundamental parameters stars : individual $(\mathrm{StH} \alpha 63)$ 


\section{Introduction}

Symbiotic stars are interacting binary systems formed by a red giant star and a hot source (in most cases a white dwarf) that ionizes the stellar wind from the cool component. They can be divided in three different infrared types, D, D' and $\mathrm{S}$, that are related to the nature of the cool giant in the system, orbital separation and physical conditions in the emitting nebulae (see Mikolajewska 1997). The number of currently identified symbiotics is much smaller than the predicted one. The catalog of Belczyński et al. (2000) contains 173 Galactic symbiotics, while the predicted number ranges from $3 \times 10^{3}$ to $4 \times 10^{5}$ (Allen 1984; Kenyon et al. 1993; Munari \& Renzini 1992; Magrini et al. 2003). Recently, several surveys have discovered new symbiotic stars increasing their number. Corradi et al. (2008, 2010) and Rodríguez-Flores et al. (2014) discovered 12 S-type and 4 D-type new symbiotics by combining the IPHAS survey and the position of the candidate stars in the 2MASS color-color diagram. Miszalski et al. (2013) used the SuperCosmos H $\alpha$ Survey (SHS) (Parker et al. 2005), selected $\mathrm{H} \alpha$ emission candidates towards the Galactic bulge, and discovered 13 S-type, 6 D-type, and 1 D'-type new symbiotics. Miszalski \& Mikolajewska (2014), also based on the SHS catalogue, found 11 S-type and 1 D-type new symbiotics in the southern hemisphere. Therefore, a total of 48 new symbiotic stars have been added to the 173 previously known ones.

Among S-type symbiotic stars, there is a group known as "yellow type symbiotics" in which the cool component is a K-type star rather than an M-type giant. Yellow symbiotics are useful objects to probe whether mass transfer has taken place in the past of these binary systems. The atmosphere of the K-type component of yellow symbiotics will be free from the strong molecular opacities due to $\mathrm{TiO}, \mathrm{CN}$ and $\mathrm{C}_{2}$ absorption features that are present in the S-type symbiotics with an M-type giant, thereby enabling the determination of stellar abundances based on atomic lines. Studies of chemical abundances in yellow symbiotics 
indeed show that these stars are enriched in s-process elements. Because these stars are not luminous enough to be self-enriched in s-process elements, the observed enrichment can be attributed to mass transfer from the former AGB star (now the white dwarf) of the system (see Smith et al 1996, 1997; Pereira \& Roig 2009). Therefore, yellow symbiotics are valuable objects to test models of AGB nucleosynthesis (Busso et al. 2001). However, they are very rare objects: only 12 Galactic yellow symbiotics are included in the catalogue of Belczyński et al. (2000) that represent only $\sim 5.4 \%$ of the total number of 221 S-type symbiotics identified to date (see above). Moreover, yellow symbiotics are members of the field Galactic halo population (Schmid \& Nussbaumer 1993; Jorissen 2003). Therefore, there is not a specific Galactic direction where they can be surveyed and, in consequence, are very difficult to find. In fact, among the $\sim 3.6$ million stellar spectra obtained with the LAMOST survey, only one new yellow symbiotic was found: LAMOST J12280490-014825.7, a K3 star with a high radial velocity and at high Galactic latitude (Li et al. 2015), similarly to some yellow symbiotics previously analyzed (Pereira \& Roig 2009).

Due to the rarity of these objects and absence of a particular Galactic location, surveys to search for new yellow symbiotics were inhibited in the past. However, Baella (2012) showed that combining their infrared properties and emission spectrum, yellow symbiotics are located in a particular region of the 2MASS color-color diagram. This led to the discovery of SS 383 as our first candidate yellow symbiotic that has been identified following the criteria above mentioned (Baella et al. 2013, hereafter BPM13).

In this work we report further spectroscopic results of our survey specifically dedicated to search and to identify new yellow symbiotics. Our major result is the identification of StH $\alpha 63$ as a new yellow symbiotic star. 


\section{Target selection}

The selection of the candidates followed the same method as we applied for the identification of SS 383 as a new candidate yellow symbiotic star (BPM13). Basically our method rely on the two observed properties of the yellow symbiotic stars, the emission-line spectrum and the K-type continuum. It was already well stablished that symbiotics

occupy particular regions in the 2MASS $(J-H)$ versus $\left(H-K_{s}\right)$ diagram (Phillips 2007; Corradi et al. 2008, 2010). Furthermore, Baella (2012) showed that, after reddening correction, yellow symbiotics are clustered around $\left(H-K_{s}\right) \sim 0.2$ and $(J-H) \sim 0.8$ because their positions in the two color diagram are mainly determined by the K-type component and they are clearly separated from S-type symbiotics with M-type components. By using the emission line catalogs of Stephenson \& Sanduleak (1977), Stephenson (1986), Schwartz, Persson \& Hamamm (1990) and Kohoutek \& Wehmeyer (1997) we have identified five more stars that are located in the region of yellow symbiotics of the 2MASS color-color diagram and, therefore, are good candidates to be yellow symbiotics. Table 1 lists the candidates and Figure 1 show their position in the $(J-H)$ versus $\left(H-K_{s}\right)$ diagram. To determine the corresponding reddening value for each object, we used the Galactic Dust Reddening and Extinction Service of IRSA (Infrared Science Archive): http://irsa.ipac.caltech.edu/applications/DUST/ to obtain the "E(B-V) Reddening" values, and converted $\mathrm{E}(\mathrm{B}-\mathrm{V})$ to $\mathrm{A}(\mathrm{J}), \mathrm{A}(\mathrm{H})$, and $\mathrm{A}(\mathrm{Ks})$ extinctions using the relationships given by Bilir et al. (2008), which are used to draw the corresponding reddening vectors in Figure 1. In particular, for $\mathrm{StH} \alpha 63$ we used a mean $\mathrm{E}(\mathrm{B}-\mathrm{V})=0.14$ (Schlafly \& Finkbeiner 2011; Schlegel et al. 1998), and obtained $\mathrm{A}(\mathrm{J}-\mathrm{H})=0.05$ and $\mathrm{A}(\mathrm{H}-\mathrm{Ks})=0.03$. 


\section{Observations \& Reductions}

Spectroscopic observations of the five targets in Table 1 were performed on 2013 and 2014 with the Cassegrain Twin Spectrograph (TWIN) attached to the $3.5 \mathrm{~m}$ telescope of the Calar Alto Observatory (Spain), and with the Goodman spectrograph attached to the $4.1 \mathrm{~m}$ telescope of the SOAR Observatory (Chile). Table 1 also includes the date of observations and the used spectrograph. TWIN includes two separate spectroscopic channels (blue and red) behind the common entrance slit aperture. The detectors were a SITe CCD 22b in the blue channel and a SITe CCD 20b in the red channel. We used gratings T08 (blue) and T04 (red) to cover the spectral ranges $3200-5800 \AA$ and $5500-7600 \AA$, respectively, at a spectral resolution of $1.08 \AA$ pixel $^{-1}$ in both channels. The slit was oriented east-west and its width was $2^{\prime \prime}$. The Goodman spectrograph was used with the Farchild CCD 486 and three gratings: blue, mid, and red mode, covering the spectral ranges 3550-6300 ,

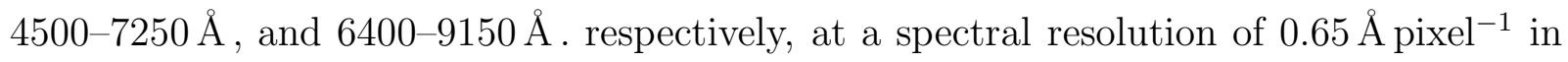
the three spectral ranges. The slit was oriented north-south and its width was $1^{\prime \prime}$. Spectra of each target were obtained with several exposure times between 15 and 1200 seconds, the short exposures to avoid saturation of possibly strong emission lines (e.g., H $\alpha$ ), the long exposures to detected faint emission lines.

The spectra were reduced using standard IRAF tasks, from bias subtraction and flat-field correction, through spectral extraction and wavelength and flux calibration. Spectrophotometric standards from Massey et al. (1988) and Hamuy et al. (1994) were also observed with the same instrumental configuration before and after the objects for flux calibration. 


\section{StH $\alpha 63$ : a new yellow symbiotic star}

Figure 2 presents the spectrum of StH $\alpha 63$ and Table 2 lists the observed fluxes of the identified emission lines. The spectrum of $\mathrm{StH} \alpha 63$ shows recombination Balmer, He I, and He II $\lambda 4686$ emission lines, and forbidden [O III] $\lambda 4363,4959,5007$ emission lines. The absorption spectrum presents the $\mathrm{TiO}$ bands at 6200, 7125, and $7160 \AA$, and absorption lines due to Fe I at $4202,5227,5269,5227,5415 \AA$, Ca I at $4226 \AA$, Mg I at $5183 \AA$, Cr I at

$5206 \AA$, Na I at $5890 \AA$, and a strong absorption around $6500 \AA$ that is due to contributions of several strong absorption lines of Fe I at $6494,6496 \AA$, Ba II at $6497 \AA$, and Ca I at $6499 \AA$. The spectrum clearly points to a symbiotic nature for StH $\alpha 63$. Moreover, the spectrum is very similar to that of the yellow symbiotic CD- $43^{\circ} 14304$ with a K7 spectral type (Schmid \& Nussbaumer 1993; Pereira et al. 1999; Mürset \& Schmid 1999), and to that of the candidate yellow symbiotic SS 383 with a spectral type K7-M0 (BPM13). These similarities strongly suggest a K spectral type for $\mathrm{StH} \alpha 63$ and, in consequence, that $\mathrm{StH} \alpha 63$ is a yellow symbiotic.

To obtain a more precise estimate for the spectral type of $\mathrm{StH} \alpha 63$ we proceeded as we did for SS 383: we measure the quantitative TiO indexes (Kenyon \& Fernández-Castro 1987) and put $\mathrm{StH} \alpha 63$ in the $[\mathrm{TiO}]_{1}-[\mathrm{TiO}]_{2}$ diagram. Figure 3 shows the position of StH $\alpha 63$ in this diagram, which lies between those of AG Dra and TV CVn, two stars with spectral types K4 III and K5.3 III, respectively (Kenyon \& Fernández-Castro 1987). Therefore, we estimate a spectral type between K4 and K6 for StH $\alpha 63$ that confirms the yellow symbiotic nature of the object.

Some physical parameters of StH $\alpha 63$, such as the color excess $E(B-V)$, optical depth in $\mathrm{H} \alpha\left(\tau_{H \alpha}\right)$, electron density $\left(N_{e}\right)$, and infrared type based on He I line intensity ratios can be obtained directly from the observed spectrum. To obtain these parameters we follow the same basic procedures as outlined in BPM13 (see also Gutiérrez-Moreno \& Moreno 
1996, and Proga et al. 1994). Table 3 shows the results. The electron density derived from the [O III] emission lines intensity ratio is $\log N_{e} \sim 8.4$ (see Table 3), a value that is typical of S-type symbiotics $\left(\log N_{e} \sim 8-10\right)$ but larger than the values found in D-type symbiotics $\left(\log N_{e} \sim 6-7\right.$, e.g., Schmid \& Schild 1990; Mikolajeska \& Kenyon 1992). As for the infrared (D or S) type (Proga et al. 1994), the line ratios $I(H e \mathrm{I} \lambda 6678) /(H e \mathrm{I} \lambda 5876)$ and $I(H e \mathrm{I} \lambda 7065) /(H e \mathrm{I} \lambda 5876)$ corrected for reddening are 0.31 and 0.78 , respectively. The value of the $I(H e \mathrm{I} \lambda 6678) /(H e \mathrm{I} \lambda 5876)$ ratio is not typical for S-type symbiotics according to Proga et al. (1994), although PU Vul investigated in their sample also presents a similar ratio of 0.30 . The $I(H e \mathrm{I} \lambda 7065) /(H e \mathrm{I} \lambda 5876)$ intensity ratio in $\mathrm{StH} \alpha 63$ is similar to that observed in the yellow symbiotic AG Dra (Proga et al. 1994).

Finally, our spectrum allow us to analyze the position of $\mathrm{StH} \alpha 63$ in the $[\mathrm{O}$ III $] 5007 / \mathrm{H} \beta$ versus [O III] $4363 / \mathrm{H} \gamma$ diagnostic diagram that was previously used in our studies of planetary nebulae (Pereira \& Miranda 2005; Pereira et al. 2010) and of the yellow symbiotic SS 383 (BPM13). The line intensity ratios (see Table 2) place $\mathrm{StH} \alpha 63$ in the region of the diagram occupied by S-type symbiotics (see BPM13, their Figure 5 ) thus further reinforcing our conclusion of the symbiotic nature for this object.

\section{The spectra of [KW97] 37-26, [KW97] 61-27, StH $\alpha 116$ and SS 360.}

Figure 4 presents the spectra of the four candidates in Table 1 that were rejected as symbiotics either due to the absence of emission lines or, when present, because they were not the appropriate ones to classify the star as a symbiotic. Noticeably, [KW97] 37-26, [KW97] 61-27, and $\mathrm{StH} \alpha 116$ do not show the $\mathrm{H} \alpha$ emission line even though the are included in $\mathrm{H} \alpha$ emission-line star catalogs (see MacConnel 1981; Stephenson 1986; Kohoutek \& Wehmeyer 1999). In the cases of [KW97] 61-27 and StH $\alpha 116$ we note that these two stars are characterized by very strong $\mathrm{TiO}$ absorption bands (Figure 4 ) that produce an 
"apparent emission peak" around $6540 \AA$, bluewards the position of $\mathrm{H} \alpha$, which may be confused with the $\mathrm{H} \alpha$ emission line in very low resolution (objective-prism) spectra. Only SS 360, discovered as an emission-line star by Stephenson \& Sanduleak (1977), presents

Balmer emission lines. However, the other typical emission lines of symbiotics (e.g., [O III]) are absent, discarding a symbiotic classification.

The spectrum of [KW97] 37-26 $\left(=[\mathrm{M} 81] \mathrm{I}-734=\mathrm{BD}+00^{\circ} 4203\right)$ is very similar to those of HD 1069 and HD 26946 with K2 I and K3 III spectral types, respectively (Jacoby et al. 1984), if we apply to these two stars a reddening of 1.4 as used in Figure 1 for [KW97] 37-26. This points out that [KW97] 37-26 is an early K supergiant or giant star. A more precise classification is not possible because our spectra do not cover the oxygen line at $7774 \AA$, the calcium infrared triplet and/or the $\mathrm{CN}$ band at $7925 \AA$ that are useful spectral features to discriminate among supergiants, giants and dwarf stars (see Pereira \& Miranda 2007). The spectra of $\mathrm{StH} \alpha 116\left(=\mathrm{BD}+14^{\circ} 2953\right)$ and [KW97] 61-27 are similar to that of SAO 21753, a K7 III star, whereas the spectrum of SS 360 is similarities to that of the M3 III star SAO 63340 (see Jacoby et al. 1984 for the spectra of the used comparison stars).

\section{The W34-index from WISE bands as an additional constraint to search for candidates to S-type yellow symbiotic stars.}

As shown above, SS 360 fullfils the two criteria used by us to select candidate yellow symbiotics but its spectrum rules out this classification. This suggests to look for additional constraints for a better selection of candidates. We have explored the data provided in the WISE (Wide-field Infrared Survey Explorer) archive. The WISE satellite scanned the whole sky in the $3.4,4.6,12$, and $22 \mu \mathrm{m}$ bands (Wright et al. 2010) commonly named W1, W2, W3 and W4, respectively. We used the W3-W4 (W34) index and created five histograms to analyze the possibility of distinguishing true S-type yellow symbiotics stars among the 
candidate objects in Figure 1.

Figure 5 shows these five histograms. Histogram A presents a sample of about 340 K-giant stars with effective temperature between 3700 and $5200 \mathrm{~K}$ (Hekker \& Meléndez 2007). It shows that the probability of finding K-giant stars with W34 $>0.4$ is very low. Histogram B presents the distribution of S-type symbiotic stars from Belczynski et al. (2000). These objects cover the entire range of W34 values in the figure, although there is a small increase of the number of S-type symbiotics in the W34 0.4-0.8 range. This could be an indication that S-type symbiotics have an emission excess between $12 \mu \mathrm{m}$ and $22 \mu \mathrm{m}$. To check this possibility, histogram C shows a sub-sample of objects of histogram B, namely, those S-type symbiotics that contain giant stars with effective temperatures higher than $3500 \mathrm{~K}$; these symbiotics present W34 values higher than 0.4. It is worth noting that effective temperatures higher than $\sim 3500 \mathrm{~K}$ are required for yellow symbiotics. Histogram D shows a sample of nine true S-type yellow symbiotics. Remarkably, they all have W34 > 0.4, indicating that the difference between S-type yellow symbiotics and "normal" K-giants (without symbiotic activity) is the W34 value. This is probably due to the presence of a dust shell in true S-type yellow symbiotics. The dust shell could add an excess of emission between $12 \mu \mathrm{m}$ and $22 \mu \mathrm{m}$, that is revealed through the W34 index that appears as an useful tool to distinguish between yellow symbiotic and "normal" K-giants.

To investigate these results further, we construct the histogram $\mathrm{E}$ with the five objects of Table 1 and SS 383 (BPM13). Only SS 383 and StH $\alpha 63$ have W34 > 0.4, which is compatible with the yellow symbiotic classification for $\mathrm{StH} \alpha 63$, and provides further support for a yellow symbiotic nature of the candidate SS 383. Moreover, SS 360 has W34 0.21 and, therefore, it is not located in the region of yellow symbiotics, in agreement with its non-yellow symbiotic nature stablished above, even though it is an emission-line star and its position on the 2MASS two color diagram favors such a classification. From these findings, 
we conclude that the W34 index can be used as a useful tool to identify candidate yellow symbiotics, in combination with emission-line star catalogs and the 2MASS color-color diagram.

\section{Conclusions}

In this work we presented spectroscopic observations of five emission-line stars that are candidates for yellow symbiotics on the basis of their position in the 2MASS color-color diagram. Only one of them, $\mathrm{StH} \alpha 63$, has been positively identified as a new yellow symbiotic. Its spectrum and position in the $[\mathrm{TiO}]_{1}-[\mathrm{TiO}]_{2}$ diagram indicates a spectral type between K4 and K6. In addition, He I emission line ratios, derived electron density, and position in the $[\mathrm{O}$ III $] 5007 / \mathrm{H} \beta$ versus $[\mathrm{O}$ III $] 4363 / \mathrm{H} \gamma$ diagram provide further support for the yellow symbiotic nature of StH $\alpha 63$. The other four candidates are rejected as symbiotics because three of them do not show emission lines (although they were classified as emission-line stars) or one shows only Balmer emission lines.

Our survey for yellow symbiotics based on their particular position in the 2MASS color-color diagram and four emission-line star catalogs raised only six candidates (SS 383 and the five stars analyzed in this work). This small number is not surprising given the rarity of yellow symbiotics. Two of them (SS 383 and StH $\alpha 63$ ) have been identified as new yellow symbiotics, which provides strong support to our method of target selection. The success of this survey becomes more clear if one takes into account that three of the six selected objects were erroneously classified as emission-line stars.

We used the WISE archive to explore the possibility of distinguishing yellow symbiotic stars. We found that yellow symbiotics have a W3-W4 (W34) index $>0.4$ and are clearly separated from "normal" K-giant stars. Therefore, the W34 index is an useful tool to 
improve the selection of candidate yellow symbiotics.

We are very grateful to the staff at the Calar Alto Observatory by the excellent observations, and to David Sanmartin (SOAR) for the excellent support given during the observation of SS 360. LFM acknowledges partial support by grant AYA2011-30228-C03.01 and AYA2014-57369-C3-3-P of the Spanish MINECO, both co-funded by FEDER funds. 
Table 1: List of objects spectroscopically observed, with the equatorial coordinates, Vmagnitudes, used spectrograph, and observed infrared color-indexes.

\begin{tabular}{|l|c|c|c|c|c|c|c|}
\hline Star & Date Obs & $\alpha_{2000}$ & $\delta_{2000}$ & $\mathrm{~V}(\mathrm{GSC})^{a}$ & $\mathrm{~T} / \mathrm{G}^{c}$ & $\mathrm{~J}-\mathrm{H}^{d}$ & $\mathrm{H}-\mathrm{K}^{d}$ \\
\hline [KW97] 37-26 & 2013 Jun 26 & 192612.5 & +005520.3 & 10.0 & $\mathrm{~T}$ & 0.88 & 0.24 \\
[KW97] 61-27 & 2013 Jun 26 & 220738 & +490018.0 & 11.8 & $\mathrm{~T}$ & 0.92 & 0.26 \\
$\mathrm{SS} 360$ & 2014 Jul 18 & 181737.7 & -283705.6 & 12.3 & $\mathrm{G}$ & 0.93 & 0.25 \\
$\mathrm{StH} \alpha 63$ & 2013 Oct 15 & 075805.9 & -074355.5 & $13.2^{b}$ & $\mathrm{~T}$ & 0.80 & 0.25 \\
$\mathrm{StH} \alpha 116$ & 2013 Jun 26 & 155219.7 & +141519.8 & 10.4 & $\mathrm{~T}$ & 0.79 & 0.22 \\
\hline
\end{tabular}

a: The Guide Star Catalog, Lasker et al. (2008)

b: Nomad Catalog, Zacharias et al. (2005)

c: T: Twin spectrograph; G: Goodmann spectrograph

d: Cutri et al. (2003) 
Table 2: Observed emission line fluxes (relative to $\mathrm{F}(\mathrm{H} \beta)=100$ ) in $\mathrm{StH} \alpha 63$.

\begin{tabular}{|c|c|c|}
\hline Wavelength $(\AA)$ & Identification & $\mathrm{F}(\lambda)$ \\
\hline 3869 & {$[\mathrm{Ne}$ III $]$} & 7.8 \\
\hline 3889 & He I & 6.3 \\
\hline 3970 & $\mathrm{H} \epsilon$ & 7.5 \\
\hline 4101 & $\mathrm{H} \delta$ & 13.9 \\
\hline 4340 & $\mathrm{H} \gamma$ & 29.6 \\
\hline 4363 & {$[\mathrm{O}$ III $]$} & 17.0 \\
\hline 4471 & He I & 5.0 \\
\hline 4686 & He II & 6.6 \\
\hline 4861 & $\mathrm{H} \beta$ & 100.0 \\
\hline 4959 & {$[\mathrm{O}$ III $]$} & 6.6 \\
\hline 5007 & {$[\mathrm{O}$ III $]$} & 25.3 \\
\hline 5876 & He I & 16.2 \\
\hline 6563 & $\mathrm{H} \alpha$ & 596.0 \\
\hline 6678 & He I & 7.4 \\
\hline 7065 & He I & 20.2 \\
\hline $\mathrm{F}(\mathrm{H} \beta) \operatorname{erg} \mathrm{cm}^{-2} \mathrm{~s}^{-1}$ & $5.1 \times 10^{-14}$ & \\
\hline
\end{tabular}


Table 3: Physical parameters of StH $\alpha 63$.

\begin{tabular}{cc}
\hline \hline Parameter & Value \\
\hline $\mathrm{E}(\mathrm{B}-\mathrm{V})$ & $0.19 \pm 0.04$ \\
$\mathrm{~A}_{V}$ & $0.59 \pm 0.12^{a}$ \\
$\tau_{H \alpha}$ & $1.1 \pm 0.1$ \\
$\log \left(\mathrm{N}_{e}\right)^{b} \mathrm{~cm}^{-3}$ & 8.4 \\
$\mathrm{He} \mathrm{I} \lambda 6678 / \lambda 5876^{c}$ & 0.31 \\
He I $\lambda 7065 / \lambda 5876^{c}$ & 0.78 \\
Spectral Type $^{d}$ & $\mathrm{~K} 4-\mathrm{K} 6$ \\
\hline \multicolumn{2}{c}{${ }^{a}$ Using $\mathrm{R}=3.1^{2}$}
\end{tabular}

${ }^{b}$ From the $\left[\mathrm{O}\right.$ III] emission lines and $\mathrm{T}_{e}=10000 \mathrm{~K}$

${ }^{c}$ Reddening corrected

${ }^{d}$ From $[\mathrm{TiO}]_{1}$ and $[\mathrm{TiO}]_{2}$ indexes 


\section{REFERENCES}

Allen, D.A., 1984, PASA, 5, 369.

Baella, N.O., 2012, PhD thesis, Observatório Nacional, Rio de Janeiro.

Baella, N.O., Pereira, C.B. \& Miranda, L.F., 2013, AJ, 146, 115 (BPM13)

Belczyński, K., Mikolajewska, J., Munari, U., Ivison, R.J. \& Friedjung, M., 2000, Astron. Astrophys. Sup. Ser., 146, 407

Bilir, S., Ak, S., Karaali, S., Cabrera-Lavers, A., Chonis, T.S. et al. 2008, MNRAS, 384, 1178.

Busso, M., Gallino, R., Lambert, D.L., Travaglio, C. \& Smith, V.V. 2001, ApJ, 557, 802

Corradi, R.L.M., Rodríguez-Flores, E.R., Mampaso, A., Greimel, R., Viironen, K. et al. 2008, A\&A, 480, 409

Corradi, R.L.M., Valentini, M., Munari, U., Drew, J.E.; Rodríguez-Flores, E.R. et al. 2010, A\&A, 509, 41

Cutri, R.M., Skrutski, M.F., van Dyk, S., Beichman, C.A., Carpenter, J.M. et al. 2003, The IRSA 2MASS All Sky Point Source Catalog, NASA/IPAC Infrared Science Archive

Downes, R.A. \& Keyes, C.D., 1988, AJ, 96, 777

Gutiérrez-Moreno, A. \& Moreno, H., 1996, PASP, 108, 972

Gutiérrez-Moreno, A., Moreno, H. \& Cortes, G., 1995, PASP, 107, 462

Gutiérrez-Moreno, A., Moreno, H. \& Costa, E., 1999, PASP, 111, 571

Hamuy, M., Suntzeff, N.B., Heathcote, S.R., Walker, A.R., Gigoux, P. et al, 1994, PASP, 106,566 
Hekker, S., \& Meléndez, J. 2007, A\&A, 475, 1003

Jacoby, G.H., Hunter, D.A. \& Christian, C.A., 1984, ApJS, 56, 257

Jorissen, A., 2003, in Symbiotic stars probing stellar evolution, ed. R.L.M. Corradi, J. Mikolajewska \& T.J. Mahoney, ASP Conf. Ser., 303, 25

Kenyon, S.J.\& Fernández-Castro, T., 1987, AJ, 93, 938

Kenyon, S.J., Livio, M., Mikolajewska, J. \& Tout, C.A., 1993, ApJ, 407, L81

Kingsburgh, R.L. \& Barlow, M.J., 1994, MNRAS, 271, 257

Kohoutek, L. \& Wehmeyer, R., 1997, Catalogue of stars in the Northern Milky Way having $\mathrm{H} \alpha$ in emission, Publisher : Hamburg Sternwarte - ESO

Lasker, B.M., Lattanzi, M.G., McLean, B.J., Bucciarelli, B., Drimmel, R., et al. 2008, ApJ, 136,735 .

Li, J., Mikolajewska, J., Chen, Xue-Fei, Luo, A.-Li, Rebassa-Mansergas, A., 2015c, RAA (Research in Astronomy and Astrophysics), 15, 1332

MacConnell, D.J., 1981, Astron. Astrophys. Sup. Ser., 44, 387

Magrini, L., Corradi, R.L.M. \& Munari, U., 2003, in Symbiotic stars probing stellar evolution, ASP Conf. Ser., 303, 539

Massey, P., Strobel, K., Barnes, J.V. \& Anderson, E., 1988, ApJ, 328, 315

Maheswar, G., Manoj, P. \& Bhatt, H.C., 2003, A\&A, 402, 963

Mikolajewska, J. \& Kenyon, S.J., 1992, AJ, 103, 579

Mikolajewska, J. 1997, in Mikolajewska J., ed., Physical Processes in Symbiotic Binaries and Related Objects. Copernicus Foundation for Polish Astronomy, Warsaw, p. 3. 
Miszalski, B., Mikolajewska, J. \& Udalski, A., 2013, MNRAS, 432, 3186

Miszalski, B. \& Mikolajewska, J., 2014, MNRAS, 440, 1410

Munari, U. \& Renzini, A., 1992, ApJ, 397, L87

Munari, U. \& Zwitter, T., 2002, A\&A, 383, 188

Mürset, U. \& Schmid, H.M., 1999, Astron. Astrophys. Sup. Ser., 137, 473

Osterbrock, D.E. \& Ferland, G.J, 2006, Astrophysics of Gaseous Nebulae and Active Galactic Nuclei (2nd ed.; Mill Valley, CA: Univ. Science Books)

Parker, Q.A., Phillipps, S., Pierce, M.J., Hartley, M., Hambly, N.C., 2005, MNRAS, 362, 689

Pereira, C.B., Baella, N.O., Daflon, S. \& Miranda, L.F., 2010, å, 509, A13

Pereira, C.B. \& Miranda, L.F., 2005, A\&A, 433, 579

Pereira, C.B. \& Miranda, L.F., 2007, A\&A, 467, 1249

Pereira, C.B. \& Roig, F., 2009, AJ, 137, 118

Phillips, J.P., 2007, MNRAS, 376, 1120

Proga, D., Mikolajewska, J. \& Kenyon, S.J., 1994, MNRAS, 268, 213

Rodríguez-Flores, E.R., Corradi, R.L.M., Mampaso, A., García-Alvarez, D., Munari, U. et al, 2014, A\&A, 567, 49

Schlafly, E.F. \& Finkbeiner, D.P., 2011, ApJ, 737, 103.

Schlegel, E.M., Finkbeiner, D.P. \& Davies, M., 1998, ApJ, 500, 525

Schmid, H.M. \& Schild, H., 1990, MNRAS, 246, 84 
Schmid, H.M. \& Nussbaumer, H., 1993, A\&A, 268, 159

Schwartz, R.D., Persson, S.E. \& Hamann, F.W., 1990, AJ, 100, 793

Smith, V.V., Cunha, K., Jorissen, A. \& Boffin, H.M.J., 1996, A\&A, 315, 179

Smith, V.V., Cunha, K., Jorissen, A. \& Boffin, H.M.J., 1997, A\&A, 324, 97

Stephenson, C.B. \& Sanduleak, N., 1977, ApJS, 33, 459

Stephenson, C.B., 1986, ApJ, 300, 779

Turnshek, D.E, Turnshek, D.A., Craine, E.R. \& Boeshaar, P.C. 1985, An Atlas of Digital Spectra of Cool Stars (Tucson: Western Research Company)

Wright, E. L. et al. 2010, AJ, 140, 1868

Zacharias, N., Monet, D. G., Levine, S. E., et al. 2005, yCat, 1297, 


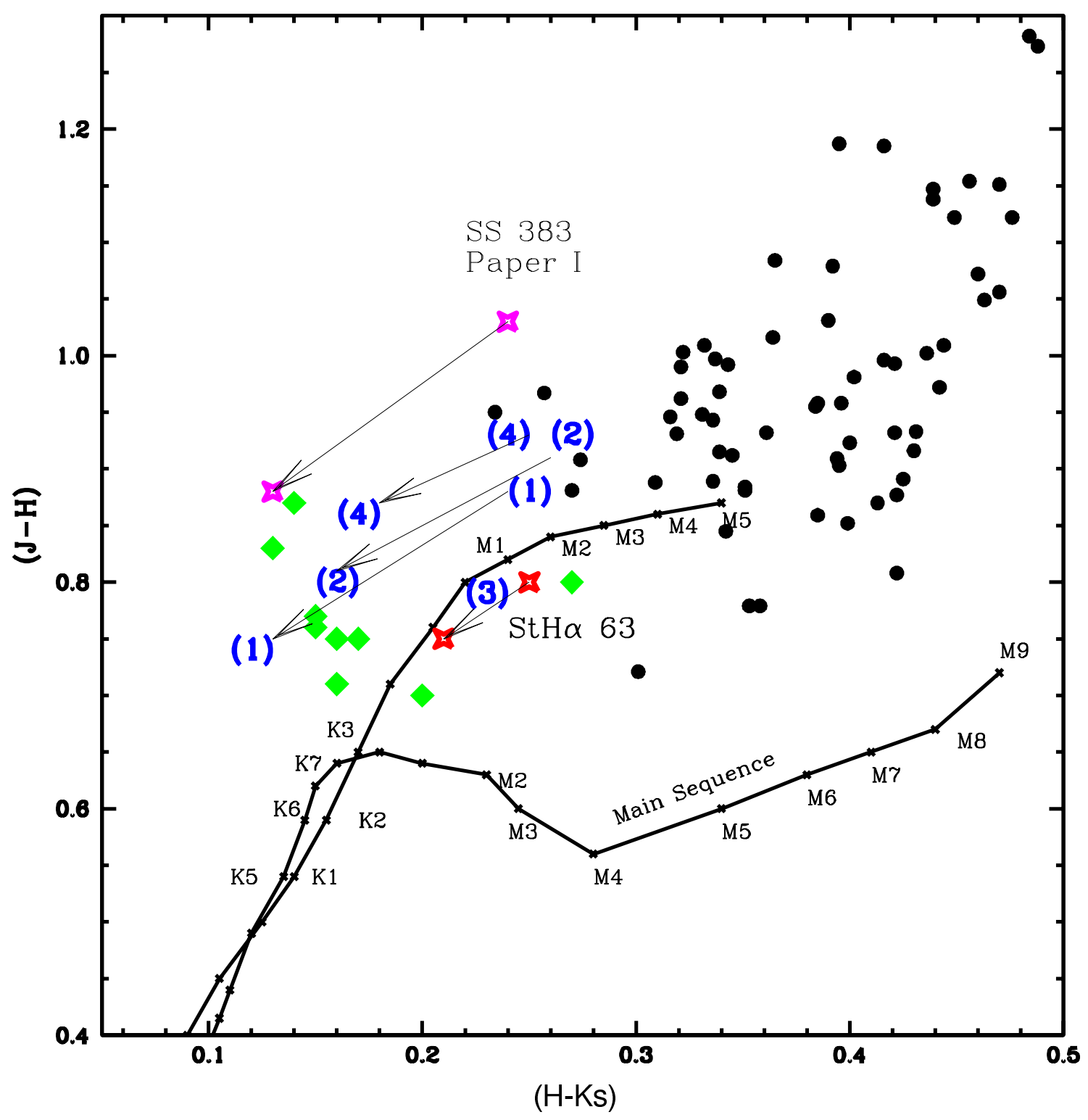

Fig. 1. - Position of the candidate yellow symbiotic stars [KW97] 37-26 (1), [KW97] 61-27 (2), StH $\alpha 116$ (3), SS 360 (4), and StH $\alpha 63$ (red star) in the 2MASS color-color diagram. The arrows connect the observed and reddening corrected $(J-H)$ and $\left(H-K_{s}\right)$ colors. For $\mathrm{StH} \alpha 116$ we do not show any correction due to the low extinction in the direction of this object. Green diamonds represent the reddening corrected infrared colors of seven S-type yellow symbiotic stars. SS 383 (magenta star) previously identified as a candidate yellow symbiotic is also shown. Black circles represent the S-type symbiotics. 

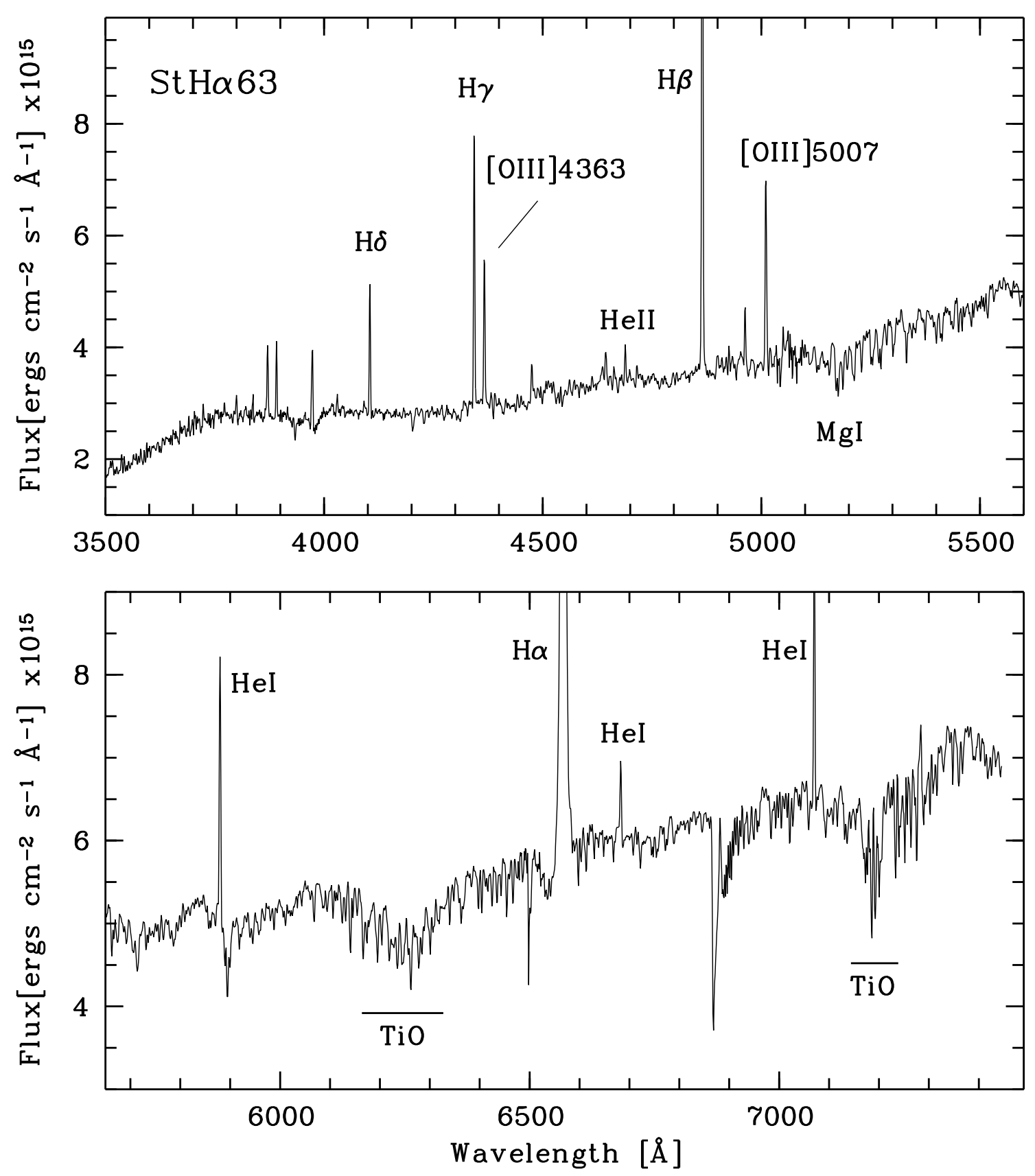

Fig. 2.- Flux calibrated spectra of $\mathrm{StH} \alpha 63$. Several emission and absorption lines, and TiO absorption bands are indicated. 


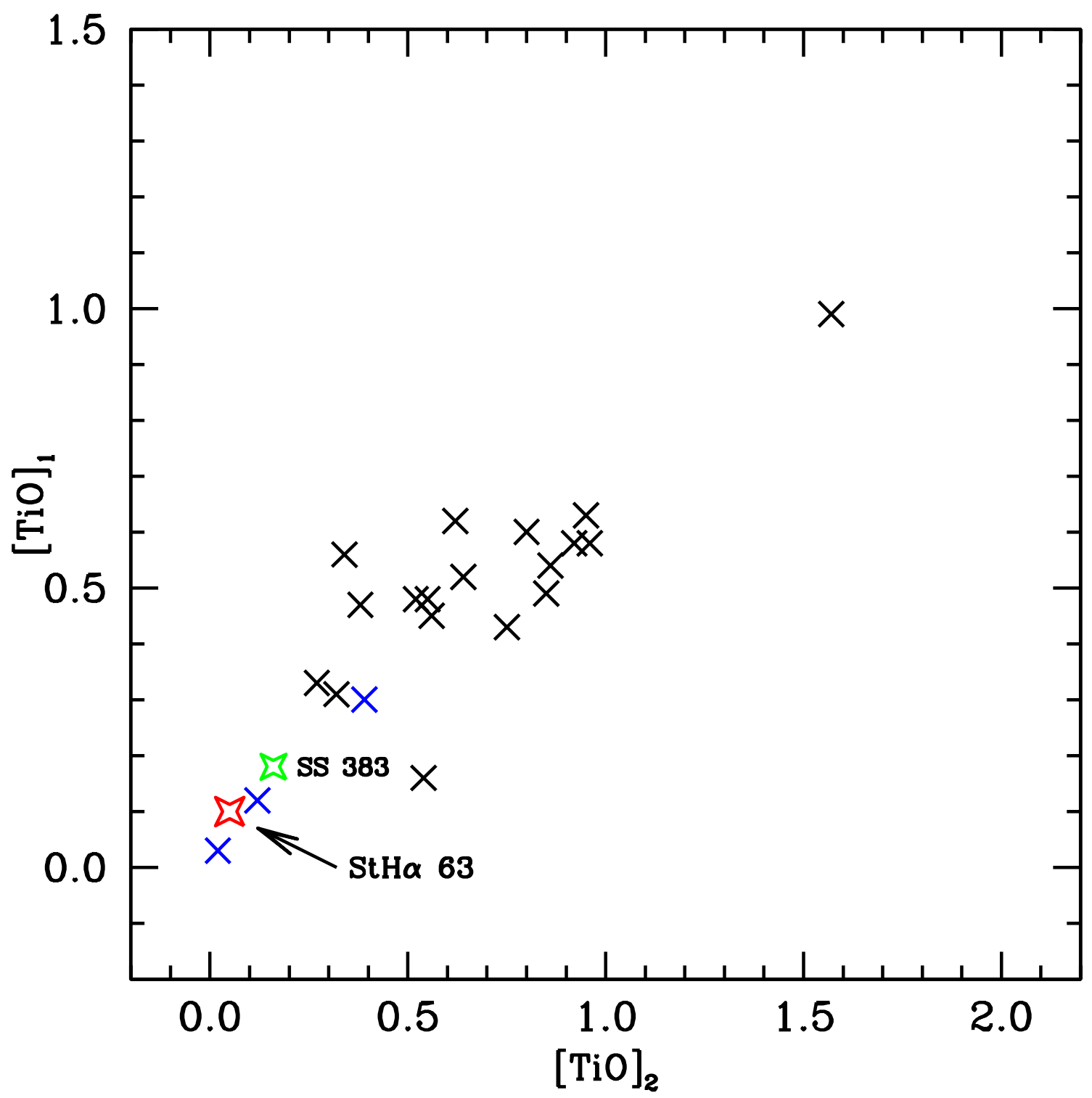

Fig. 3.- Position of $\mathrm{StH} \alpha 63$ (red star) in the $[\mathrm{TiO}]_{1}$ vs $[\mathrm{TiO}]_{2}$ diagram for a sample of symbiotic stars analyzed by Kenyon \& Fernández-Castro (1987b). Black crosses represent the S- and the D-type symbiotics with spectral types later than M0. The smaller sample of S-type symbiotics with spectral types not later than K9 (the yellow symbiotics) AG Dra, RS Oph and TX CVn also analyzed by Kenyon \& Fernandez-Castro (1987b), are represented in this diagram by red crosses. The green star represents SS 383 and the red star StH $\alpha 63$. 

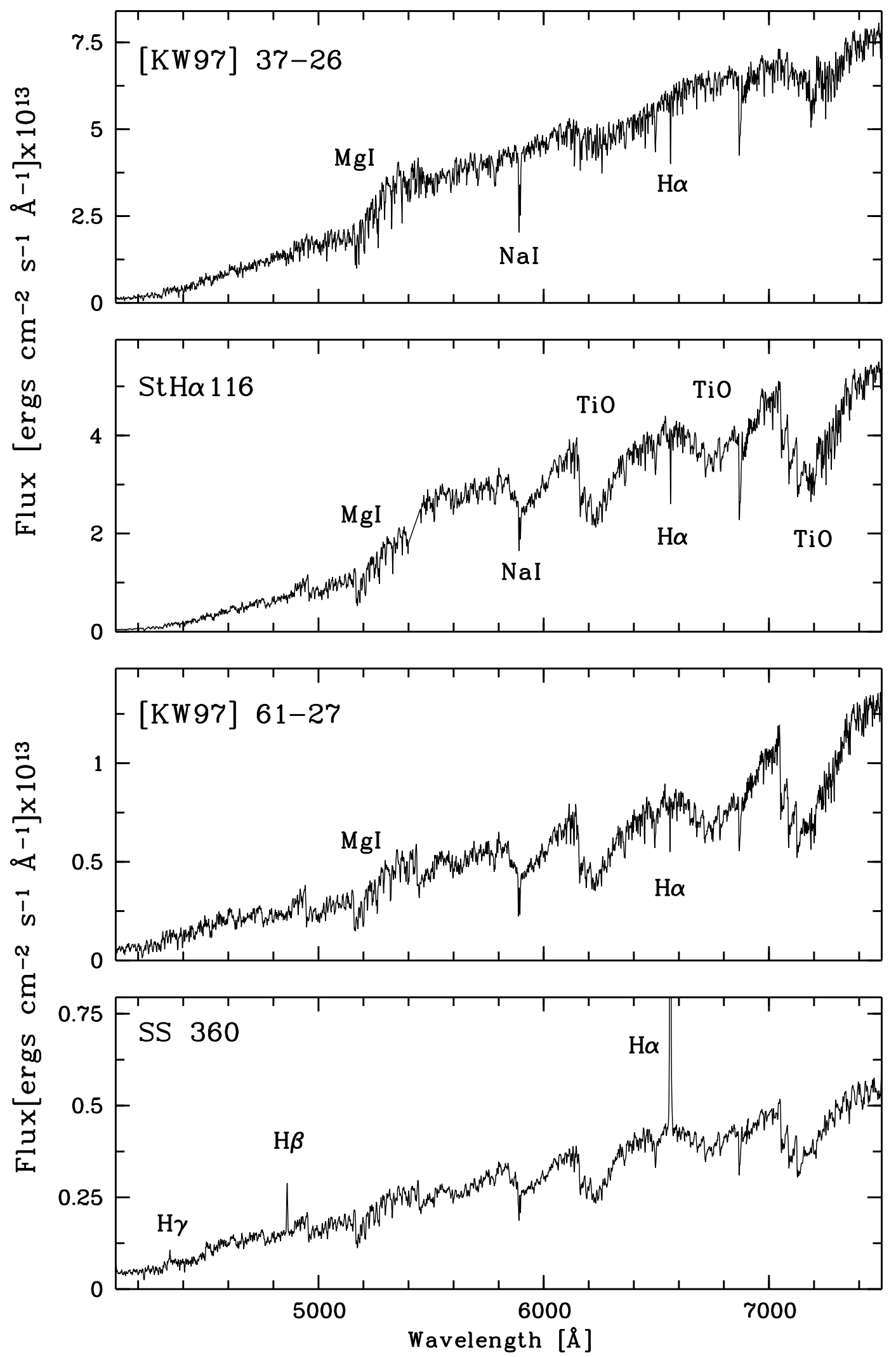

Fig. 4.- Flux calibrated spectrum of the four candidate yellow symbiotics [KW97] 37-26, StH $\alpha 116$, [KW97] 61-27, and SS 360. Some emission and absorption features are indicated. 


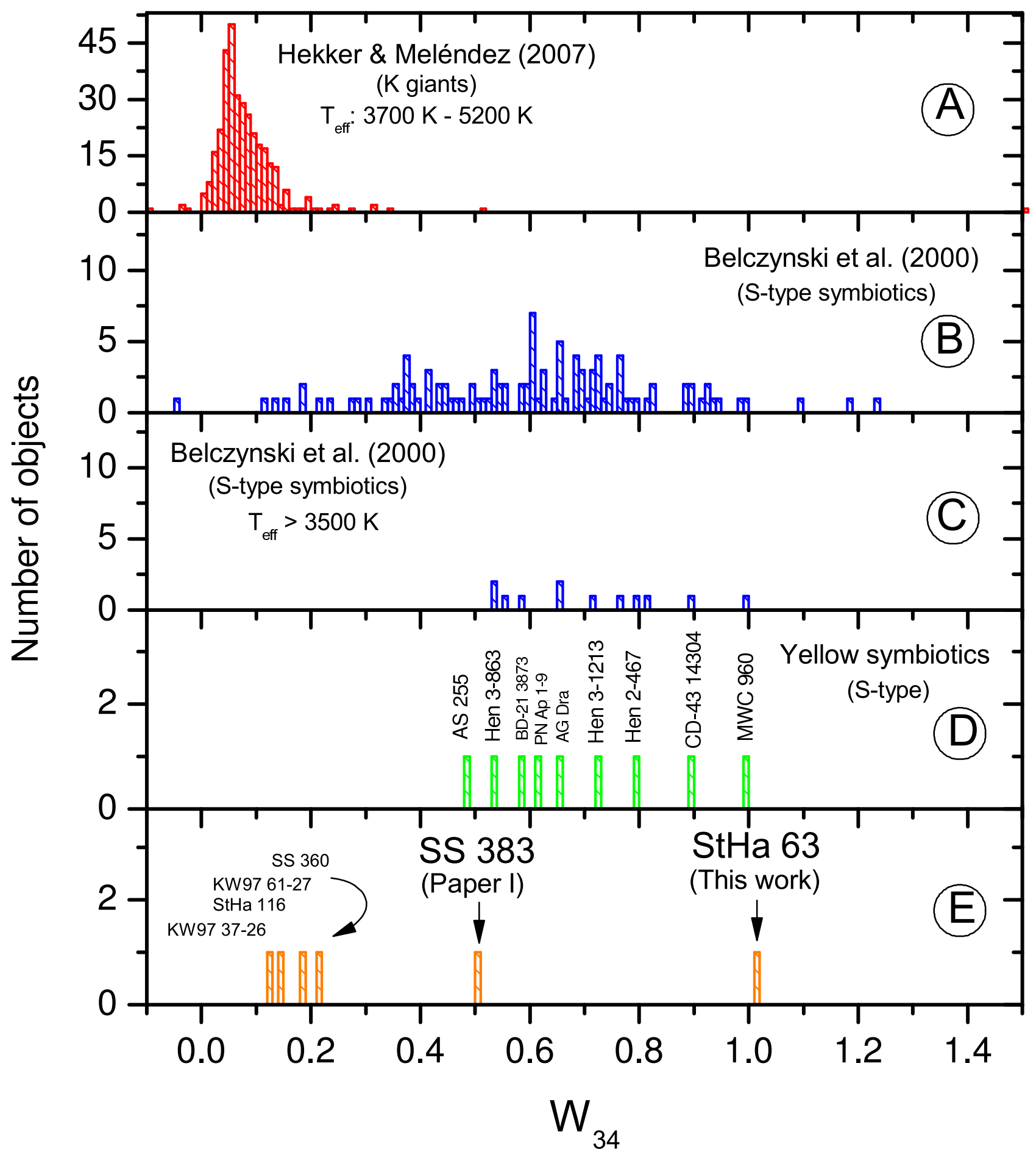

Fig. 5.- Distribution of the WISE W3-W4 (W34) index for five samples of K-giant and symbiotic stars. The content of the histograms is explained within each of them (see text for details). 